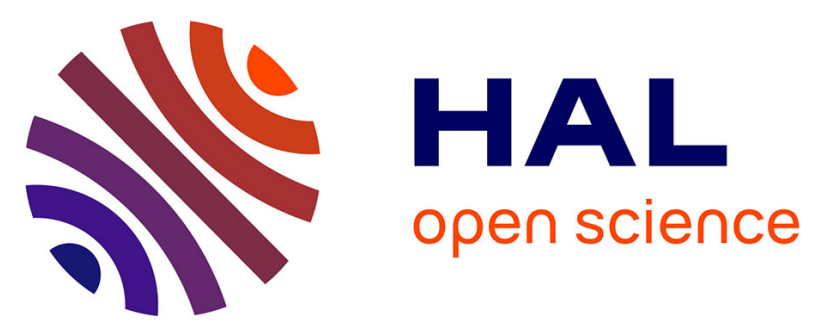

\title{
Enfants et adultes forts consommateurs de sucres libres en France: quels changements alimentaires pour respecter les recommandations nutritionnelles?
}

Mathieu M. Maillot, Lisa Privet, Sarah Vaudaine, Anne Lluch, Nicole Darmon

\section{- To cite this version:}

Mathieu M. Maillot, Lisa Privet, Sarah Vaudaine, Anne Lluch, Nicole Darmon. Enfants et adultes forts consommateurs de sucres libres en France: quels changements alimentaires pour respecter les recommandations nutritionnelles?. Cahiers de Nutrition et de Diététique, 2017, 52 (Supplément 1), pp.S66-S79. 10.1016/S0007-9960(17)30200-6 . hal-02626034

\section{HAL Id: hal-02626034 \\ https://hal.inrae.fr/hal-02626034}

Submitted on 26 May 2020

HAL is a multi-disciplinary open access archive for the deposit and dissemination of scientific research documents, whether they are published or not. The documents may come from teaching and research institutions in France or abroad, or from public or private research centers.
L'archive ouverte pluridisciplinaire HAL, est destinée au dépôt et à la diffusion de documents scientifiques de niveau recherche, publiés ou non, émanant des établissements d'enseignement et de recherche français ou étrangers, des laboratoires publics ou privés. 
3 Titre: Enfants et adultes forts consommateurs de sucres libres en France : quels

4 changements alimentaires pour respecter les recommandations nutritionnelles ?

5 Title: Characteristics of French children and adults with excessive free sugar intakes. Which dietary

6 changes are needed to meet nutritional recommendations?

7

8 Auteurs :

9 Matthieu Maillot ${ }^{*^{*}}$, Lisa Privet ${ }^{1}$, Sarah Vaudaine ${ }^{2}$, Anne Lluch ${ }^{2}$, Nicole Darmon ${ }^{3,4}$

$10{ }^{1}$ MS-Nutrition, 27 bd Jean Moulin, 13005 Marseille, France

112 Danone Nutricia Research, RD128 - Avenue de la Vauve, 91128 Palaiseau Cedex, France

$12{ }^{3}$ NORT, INRA, AMU, INSERM, 27 bd Jean Moulin, 13005 Marseille, France

${ }^{4}$ MOISA, INRA, CIHEAM-IAMM, CIRAD, Montpellier SupAgro, 2 place Pierre Viala, 34060 Montpellier,

France

15

*Auteur correspondant : matthieu.maillot@ms-nutrition.com 
L'Organisation Mondiale de la Santé (OMS) recommande une consommation de sucres libres inférieure à $10 \%$ de l'apport énergétique total chez les enfants et les adultes. L'objectif de cette étude était de décrire les caractéristiques et la diète des consommateurs dépassant cette recommandation (appelés ici forts consommateurs) en France et de modéliser les changements alimentaires nécessaires pour atteindre l'ensemble des recommandations nutritionnelles. Les apports nutritionnels de 1230 enfants et 1719 adultes ont été estimés à partir de l'enquête alimentaire française INCA2 (2006-2007) et de la base CIQUAL 2013 complétée des teneurs en sucres libres (SL). La population a été séparée en 4 classes d'âge (3-6 ans, 7-11 ans, 12-17 ans et les adultes) puis en 2 sous-populations : SL-Compliant, pour les individus respectant la recommandation en sucres libres (apport inférieur ou égal à 10\% de l'apport énergétique total), et SL-Excès, pour ceux ne la respectant pas. Dans la sous-population SL-Excès, pour chaque diète individuelle observée, une nouvelle diète iso-calorique optimisée - définie comme respectant un ensemble de 33 recommandations nutritionnelles, tout en restant le plus proche possible de la diète observée - a été modélisée par programmation linéaire. Quand aucune solution mathématique n'était possible, la diète était considérée comme non optimisable. Le pourcentage d'individus SL-Excès décroit avec l'âge : $92 \%$, 86\%, 75\%, et $41 \%$ chez les 3-6 ans, 7-11 ans, 12-17 ans et chez les adultes, respectivement. Pour tous les groupes d'âge, les SL-Excès consomment plus de sucres libres pendant les repas principaux mais surtout hors repas. Les diètes observées des SL-Excès n'étaient pas optimisables pour $40 \%, 9 \%, 11 \%$ et $0,1 \%$ chez les $3-6$ ans, 7-11 ans, les 12-17 ans et chez les adultes, respectivement. Pour les autres, l'optimisation a conduit pour toutes les tranches d'âge à diminuer les produits sucrés, les boissons sucrées et les jus de fruits, et à augmenter les quantités d'eau, de fruits et légumes, féculents, et en plus pour les 7-17 ans à 
$41 \mathrm{~g} / \mathrm{j}$, selon la classe d'âge), via une moindre contribution des produits sucrés (de $-14,3$ à $-21,3 \mathrm{~g}$ de

42 sucres libres/j) et des boissons sucrées et jus de fruits (de -6,7 à -13,4 g de sucres libres/j).

43 En conclusion, une forte majorité des enfants français a des apports excessifs en sucres libres. II est

44 généralement possible d'optimiser la diète des forts consommateurs de sucres libres, en s'éloignant

45 le moins possible de la diète observée. Néanmoins, compte tenu des contraintes imposées dans le modèle, une exploration complémentaire est nécessaire pour comprendre les raisons pour lesquelles

47 il est impossible d'atteindre une diète nutritionnellement adéquate pour $40 \%$ des enfants de 3-6 ans

48 ayant des apports excessifs en sucres libres.

49 Mots-clefs: sucre, programmation linéaire, recommandations nutritionnelles, habitudes alimentaires, snacking, France, INCA2 


\section{Abstract}

The World Health Organization (WHO) recommends reducing the intake of free sugars to less than $10 \%$ of total energy intake for both children and adults. The objective of this study was to characterize the diet of French children and adults with excessive free sugar intakes and to model the dietary changes needed to achieve all the nutritional recommendations.

The nutritional intakes of 1,230 children and 1,719 adults were estimated from the French national survey INCA2 (2006-2007) and the CIQUAL 2013 French food composition database completed with free sugars (FS). The population was divided into 4 age classes (3-6 years, 7-11 years, 12-17 years and adults) and then into 2 groups, based on the contribution of free sugars to energy intake lower or equal to $10 \%$ (FS-Acceptable) or greater than 10\% (FS-Excess). For each individual observed diet from the FS-Excess group, a new iso-caloric optimized diet - defined as complying with a set of 33 nutritional recommendations, while staying as close as possible to the diet observed - was modeled by linear programming. When no mathematical solution was possible, the diet optimization was considered unfeasible.

The percentage of FS-Excess individuals decreased with age: $92 \%, 86 \%, 75 \%$, and $41 \%$ in the $3-6$ years, 7-11 years, 12-17 years and in the adults, respectively. For all age groups, FS-Excess individuals consumed more free sugars, particularly out of the main meals. Diet optimization was unfeasible for $40 \%, 9 \%, 11 \%$ and $0.1 \%$ of FS-Excess individuals in 3-6 years, $7-11$ years, $12-17$ years and adults, respectively. When diet optimization was feasible, the main changes for all age groups were decreases in sweet products, sugar-sweetened beverages and fruit juices, and increases in water, fruit and vegetables, starchy foods. In the 7-17 years, dairy products (milk or yoghurt) were also increased. The diet optimization process decreased free sugars $(-26$ to $-30 \mathrm{~g}$ of free sugars/d, depending on the age group), via a lower contribution of sweet products (from -14.3 to $-21.3 \mathrm{~g}$ of free sugars $/ \mathrm{d}$ ) and sugar-sweetened beverages and fruit juices (-6.7 to $-13.4 \mathrm{~g}$ of free sugars $/ \mathrm{d}$ ). 
75 In conclusion, a large majority of French children have excessive intakes of free sugars. It is generally 76 possible to optimize the diet of individuals with excessive intakes of free sugars, staying as close as 77 possible to the observed diet. However, given the constraints imposed in the model, further exploration is needed to understand why it is impossible to achieve a nutritionally adequate diet for $40 \%$ of 3-6 years old children with excessive intakes of free sugars.

80 Keywords: Sugars, linear programming, nutrient recommendations, dietary habits, snacking, France, 


\section{Introduction}

83

Dans un contexte d'augmentation de la prévalence des maladies non transmissibles, les apports en sucres sont de plus en plus pointés du doigt: l'excès de sucre augmente le risque de caries [1], favorise la prise de poids [2] et a été incriminé dans la survenue et/ou les complications associées au diabète de type $2[3,4]$ et aux maladies cardiovasculaires [5-7]. De plus, une consommation élevée de sucres ajoutés a été associée à une alimentation de plus faible qualité nutritionnelle, avec notamment de faibles apports en micronutriments [8].

Pour l'Organisation Mondiale de la Santé (OMS), le terme "sucres" comprend tous les mono- et disaccharides et le terme "sucres ajoutés" fait référence aux sucres ajoutés lors des procédés industriels, culinaires ou lors de la consommation. Les "sucres libres" correspondent aux sucres ajoutés additionnés des sucres naturellement présents dans le miel, les sirops, les jus et les concentrés de fruits [9].

L'OMS considère que la lutte contre la consommation excessive de sucres est une priorité, et recommande depuis 2003 de limiter l'apport en sucres libres à moins de 10\% de l'apport énergétique (soit 50 g pour un apport calorique de $2000 \mathrm{Kcal}$ ), aussi bien chez les adultes que chez les enfants [10]. En Europe, l'Autorité européenne de sécurité des aliments (EFSA) a considéré en 2010 que les données étaient insuffisantes pour recommander un apport maximal en sucres [11]. En France, il existe une recommandation sur les sucres totaux (hors lactose et galactose), récemment proposée par l'ANSES pour les adultes et fixée à moins de $100 \mathrm{~g}$ par jour (notamment sur la base des apports en fructose) [12]. La Confédération européenne des industries agroalimentaires (CIAA) recommande un apport en sucres de 90 à $110 \mathrm{~g}$ (soit proche de la recommandation de $100 \mathrm{~g}$ de l'ANSES) pour l'étiquetage des repères nutritionnels journaliers (RNJ) en s'appuyant sur la limite de l'OMS de 10\% de l’apport énergétique pour les sucres libres [13].

Les apports en sucres, quant à eux, varient largement d'un pays à l'autre [11,14-17], selon l'âge [18] et les habitudes alimentaires (notamment les prises alimentaires en dehors des repas principaux) 

porte sur les sucres libres, les données de ces enquêtes ne permettent pas toujours faire la distinction entre les différents types de sucres. Le respect de la recommandation OMS sur les sucres libres a pu être étudiée en Australie [20], au Royaume Uni [21] et aux Pays-Bas [22], aussi bien chez les enfants et que chez les adultes.

Ces données n'existant pas pour la France, l'objectif de cette étude était d'estimer, parmi les enfants et les adultes, le pourcentage des forts consommateurs de sucres libres (c'est-à-dire les individus dont les apports en sucres libres excèdent la recommandation de l'OMS), de décrire leurs caractéristiques ainsi que de modéliser les changements alimentaires qui seraient nécessaires pour qu'ils respectent l'ensemble des recommandations nutritionnelles.

\section{Données de consommation et de composition nutritionnelle}

Les données de l'enquête alimentaire INCA2 [23] réalisée en 2006-2007 sur un échantillon représentatif de la population française, ont été utilisées pour cette étude. Les enfants et les adultes ont rempli un carnet alimentaire pendant 7 jours consécutifs. Les apports nutritionnels ont été estimés à partir des données de consommations, reliées à la base de données de composition nutritionnelle CIQUAL 2013 complétée des teneurs en sucres libres [24]. Les boissons alcoolisées ont été retirées de l'analyse. Les aliments ont été répartis en 31 sous-groupes, eux-mêmes agrégés en 9 grands groupes : "Fruits et légumes», "Féculents », "Viandes, œufs et poissons », " Plats préparés et sandwichs ", "Produits laitiers ", "Produits sucrés ", "Eaux et boissons ", " Matières grasses et sauces ", "Produits à base de soja ». Le groupe " Fruits et légumes» comprend l'ensemble des fruits oléagineux et le groupe des "Féculents " comprend les légumineuses. Les sous-groupes "laits", " yaourt ", "fruits » ont été davantage détaillés afin d'identifier ceux contenant ou non des sucres 
131 libres (par ex: "yaourts nature » versus "yaourts sucrés »). La liste complète des groupes et sous-

132 groupes d'aliments peut être retrouvée dans le supplément 3 (disponible en ligne).

133 Après suppression des sous-déclarants avec la méthode de Goldberg [25] et sélection de ceux ayant 134 déclaré 7 jours de consommation, l'échantillon d'analyse descriptive comprend1380 enfants âgés de 1353 à 17 ans répartis en 3 classes d'âge : 3-6 ans $(n=230), 7-11$ ans $(n=412)$ et 12-17 ans $(n=738)$. Pour 136 les adultes, l'échantillon d'analyse descriptive comprend 1719 adultes âgés de 20 à 75 pour rester 137 homogène à l'échantillon d'analyse ayant fait l'objet d'une publication antérieure [24]: 7 individus 138 normo-déclarants pour les lesquels la diète n'est pas optimisable (6 chez les SL-Compliants et 1 chez 139 les SL-Excès) ont été exclus de l'analyse descriptive et les adultes de 18-19 ans n'ont pas été 140 intégrés .

143 En nutrition, le principe de la modélisation par programmation linéaire est de trouver les 144 combinaisons d'aliments qui permettent de respecter un ensemble de contraintes définies en 145 fonction de l'étude, incluant généralement un ensemble de recommandations nutritionnelles [26146 29]. Dans un modèle de programmation linéaire, les variables correspondent aux quantités 147 d'aliments que le modèle pourra faire varier pour respecter un ensemble de contraintes incluant des 148 recommandations nutritionnelles (les contraintes nutritionnelles), des fourchettes de quantités 149 d'aliments et de groupes d'aliments jugées acceptables (les contraintes d'acceptabilité). Une 150 combinaison linéaire des variables (appelée fonction objectif) est ensuite choisie pour être optimisée 151 (c'est-à-dire minimisée ou maximisée).

152 Dans ce travail, une approche de modélisation individuelle de diètes [30] a été appliquée chez les 153 enfants et les adultes de façon à obtenir pour chaque diète observée (c'est-à-dire les apports 154 alimentaires déclarés comme "consommés ») une diète modélisée nutritionnellement adéquate 155 c'est-à-dire une diète optimisée construite à partir de la diète observée et respectant un ensemble 
157 observée et la diète modélisée, et cet écart a été minimisé afin de simuler une diète nutritionnelle adéquate la plus proche possible de celle de départ. Il est important de noter que cette modélisation a été réalisée pour chaque individu et à calories constantes. Les 33 contraintes nutritionnelles imposées pour la modélisation et les contraintes d’acceptabilité sont listées dans le supplément 1 (disponible en ligne). Pour les macronutriments, les recommandations de l'OMS [9] ont été utilisées, alors que les recommandations françaises [31] ont été appliquées pour les vitamines et minéraux. La limite maximale en sodium correspondait aux recommandations des pays nordiques [32]. Une contrainte sur l'apport en eau $\left(\mathrm{H}_{2} \mathrm{O}\right)$ a été ajoutée et correspond à la recommandation proposée par I'EFSA [33] qui préconise pour les enfants de 3 ans : $1,3 \mathrm{~L} / \mathrm{j}$; pour les enfants de $4-8$ ans : $1,6 \mathrm{~L} / \mathrm{j}$; pour les enfants de $9-13$ ans : 2,1L/j pour les garçons et 1,9L/j pour les filles; chez les sujets de plus de 14 ans : $2 \mathrm{~L} / \mathrm{j}$ pour les femmes et $2,5 \mathrm{~L} / \mathrm{j}$ pour les hommes.

Le détail des contraintes du modèle utilisé est disponible dans 2 publications précédentes sur les adultes [24,34]. Cette étude est la première à appliquer la modélisation individuelle aux diètes d'enfants dans l'enquête INCA2.

\section{Analyse statistique}

172 Au sein de chacune des classes d'âge, l'échantillon a été séparé en 2 sous-populations: SL-

173 Compliants, pour les individus respectant la recommandation OMS en sucres libres (apport inférieur 174 ou égal à $10 \%$ de l'apport énergétique total), et SL-Excès, pour ceux ne la respectant pas.

175 Les caractéristiques sociodémographiques et anthropométriques, le temps moyen passé devant la télévision et l'ordinateur (sédentarité), la fréquence de consommations hors repas obtenue à partir

177 d'un questionnaire, ainsi que les consommations alimentaires et la qualité nutritionnelle des diètes 178 observées ont été comparées entre les populations SL-Compliants et SL-Excès. La qualité 179 nutritionnelle a été estimée par les apports en macro et micronutriments mais aussi à l'aide 180 d'indicateurs synthétiques tels que le MAR (\%/j) (Mean Adequacy Ratio) [35] et le MER (\%/j) (Mean 
Excess Ratio) [36] pour les enfants et les adultes. Une diète de bonne qualité nutritionnelle est

182 caractérisée par un MAR élevé et un MER bas. Les apports moyens en énergie et en sucres libres ont

183 été estimés séparément selon qu'ils provenaient des trois repas principaux ou des prises alimentaires hors repas.

185

186

187

188

189

190

191

192

193

194

195

196

197

198

199

200

201

202

203

204

Les changements alimentaires nécessaires pour atteindre l'adéquation nutritionnelle ont été étudiés uniquement chez les SL-Excès. II a été choisi dans ce modèle de limiter l'augmentation des produits enrichis en vitamines et minéraux (comme les céréales du petit déjeuner et les laits de croissance) lorsqu'ils étaient initialement consommés et également, et de ne pas permettre leur introduction dans les diètes optimisées lorsqu'ils n'étaient pas initialement consommés par l'individu. Cette limitation du recours aux produits enrichis (et aux suppléments) est le choix qui est généralement fait dans les optimisations par programmation linéaire: en effet, il s'agit d'éviter que les modèles s'engouffrent vers une solution de facilité qui serait celle de l'ajout systématique de produits enrichis, afin de pouvoir réellement identifier quels aliments sont à même de couvrir les écarts entre les apports observés et les niveaux imposés par les contraintes nutritionnelles. Au cours de la modélisation des diètes individuelles, il a été impossible de trouver une optimisation pour 1 adulte (soit $0,1 \%$ de l'échantillon des SL-Excès) et 175 enfants ( $n=85$, soit $40 \%$ des 210 SL-Excès de 3-6 ans; $\mathrm{n}=31$ soit $9 \%$ des 353 SL-Excès de $7-11$ ans; et $n=59$ soit $11 \%$ des 551 SL-Excès de 12 -17ans). Cette infaisabilité était due à l'incompatibilité entre les contraintes nutritionnelles (dont celle sur l'énergie), les contraintes d'acceptabilité et le répertoire alimentaire de ces individus. Le souséchantillon d'analyse des changements alimentaires porte sur les individus SL-Excès avec une diète faisable (SL-Excès-optimisables) et comprend 699 adultes et 939 enfants ( $n=125$ de 3-6 ans, $n=322$ de 7-11 ans, $n=492$ de 12-17 ans). Dans chaque classe d'âge, les changements alimentaires ont été analysés par groupe et sous-groupes d'aliments. Les variations des groupes et sous-groupes d'aliments ont été comparées à zéro (zéro correspondant à une absence de variation). Les contributions des différents groupes d'aliments aux différents types de sucres (totaux, libres, non libres) ont été comparées entre les diètes observées et modélisées. Plus spécifiquement, les 
différents types de produits laitiers (laits et yaourts, nature ou sucrés, et les fromages) ont été analysés dans les diètes observées et modélisées.

Toutes les moyennes et tests statistiques ont pris en compte la méthode d'échantillonnage d'INCA2 ainsi que les pondérations nécessaires à la représentativité des populations. Les analyses ont été réalisées séparément dans chacune des 4 classes d'âge.

Le logiciel SAS 9.4 a été utilisé pour la modélisation de diètes et les analyses statistiques. Le seuil de significativité des tests statistiques utilisant le modèle linéaire général (GLM) était de 5\%. l'objet d'une publication [24].

\section{Résultats et discussion}

\section{Apport moyen en sucres libres et pourcentage d'individus en excès}

219 L'apport en sucres libres représente en moyenne respectivement 14,7\% et 9,5\% de l'apport énergétique chez les enfants (3-17 ans) et les adultes (20-75 ans) français (figure 1A). Chez les adultes, cet apport moyen ne prend pas en compte les boissons alcoolisées, dont les apports en sucres libres contribuent à seulement 0,16\% de l’apport énergétique total (résultats non montrés).

223 Le pourcentage de consommateurs de sucres libres en excès (SL-Excès) est de 83\% chez les enfants et 224 41\% chez les adultes, et est décroissant avec l'âge (de 92\% chez les 3-6 ans à 21\% chez les 65-75 ans, figure 1B). Le fort pourcentage de consommateurs de sucres libres en excès chez les enfants s'explique par des apports en sucres libres (en $\mathrm{g} / \mathrm{j}$ ) proches de ceux des adultes malgré des apports énergétiques plus faibles (résultats non montrés). 
231 libres des enfants est équivalente à celle des enfants français, alors qu'ils sont plus faibles en

232 Australie (12\%), où le pourcentage de consommateurs de sucres libres en excès est estimé à environ 70\%-75\% chez les enfants et 45-60\% chez les adultes (avec également un effet décroissant avec l'âge) [20].

La répartition par sexe est équivalente entre les SL-Compliants et SL-Excès quel que soit l'âge, excepté pour les 3-6 ans où les SL-Compliants sont plus souvent des garçons (tableau 1). Chez les adultes, les SL-Compliants sont âgés de 10 ans de plus en moyenne que les SL-Excès. Ces derniers déclarent un temps moyen de sédentarité plus élevé (221 min/j) que les SL-Compliants (195 min/j), alors qu'aucune différence n'est trouvée dans les autres tranches d'âge. Chez les adolescents (12-17 ans) et les adultes seulement, les SL-Compliants sont plus souvent en surpoids ou obèses $(23,9 \%$ et $47,8 \%$ respectivement) que les SL-Excès (10,4\% et $29 \%$ respectivement), et ils déclarent une plus faible fréquence de consommations hors repas que les SL-Excès $(24,2 \%$ vs $41,9 \%$ chez les adolescents, et $13,1 \%$ vs $23,8 \%$ chez les adultes déclarant au moins 2 consommations hors repas par jour). A noter que bien que ces résultats soient non significatifs chez les 7-11 ans, les SL-Compliants sont également plus souvent en surpoids ou obèses $(39,1 \%)$ que les SL-Excès $(16,3 \%)$. Chez les 3-6 ans, aucune différence n'est observée. Ces résultats contre-intuitifs (proportionnellement moins de personnes en surpoids ou obèses chez des forts consommateurs de sucres libres) ont déjà été constatés dans d'autres études transversales, et pourraient s'expliquer par une sous-déclaration ou par le caractère transversal des études. 
Le point commun à toutes les tranches d'âge est un apport énergétique hors des repas principaux

255 plus important chez les SL-Excès que les SL-Compliants (de $+75 \mathrm{kcal} / \mathrm{j}$ à $171 \mathrm{kcal} / \mathrm{j}$ selon la tranche

256 d'âge, tableau 2). Chez les enfants, l’apport énergétique des consommations hors repas provient

257 principalement de prises alimentaires entre le déjeuner et le diner (supplément 2 disponible en

ligne) ; l'analyse n'a pas été faite chez les adultes. Quant à l'apport énergétique journalier, il est équivalent entre les SL-Compliants et les SL-Excès chez les enfants de 3-6 ans et 7-11 ans alors que chez les 12-17 ans et les adultes, il est plus élevé chez les SL-Excès par rapport aux SL-Compliants

(tableau 2). La méthodologie d'INCA2 ne permet pas d'attribuer la totalité de la prise alimentaire entre le déjeuner et le diner au moment spécifique du goûter mais ces résultats suggèrent que les enfants SL-Excès seraient des plus grands consommateurs de goûters, moment où la prise alimentaire est principalement composée de produits au goût sucré [38].

265 Concernant les sucres libres, dans toutes les tranches d'âge et à tous les moments de consommation, les SL-Excès consomment plus de sucres libres que les SL-Compliants (de $+31,4 \mathrm{~g} / \mathrm{j}$ à $+45,2 \mathrm{~g} / \mathrm{jour}$ selon les tranches d'âges) notamment lors des consommations hors repas (34 à $46 \%$ des apports supplémentaires en sucres libres) (tableau 2).

Globalement, les SL-Excès ont une alimentation plus dense en énergie que les SL-Compliants (7-11 ans : 193 vs $178 \mathrm{Kcal} / 100 \mathrm{~g}, 12-17$ ans : 198 vs $182 \mathrm{Kcal} / 100 \mathrm{~g},>20 \mathrm{ans}: 185$ vs $165 \mathrm{Kcal} / 100 \mathrm{~g})$, excepté

27219 points, expliqué par les apports plus élevés en sucres libres (tableau 2). Chez les 12-17ans, les SL-

273 Excès ont un MAR plus élevé (reflet d'une meilleure qualité nutritionnelle) que les SL-Compliants 274 (78,9\% vs 76,7\% /j) ce qui peut s'expliquer par un apport énergétique plus élevé de $215 \mathrm{kcal} / \mathrm{j}$ chez les 275 SL-Excès. Si le MAR avait été calculé pour 2000kcal, la densité nutritionnelle des diètes des SL276 Compliants aurait été supérieure à celle des SL-Excès, comme dans une étude au Royaume-Uni 277 [21].Chez les adultes français de notre étude, les SL-Compliants présentent un MAR plus élevé que 
les SL-Excès $(84,8$ vs $82,4 \% / j)$. Ces résultats sont en accord avec ceux d'une revue récente qui montre que les apports en sucres sont associés à une plus faible qualité nutritionnelle des diètes [8].

Dans la diète des SL-Excès, les sucres libres sont apportés principalement par les produits sucrés (de $30,7 \%$ à $53,9 \%$ des apports totaux selon la classe d'âge) et les boissons (de $18,6 \%$ à $33,3 \%$ des Braesco ce numéro [39].

II a été possible de construire une diète optimisée pour la grande majorité des individus SL-Excès, sauf pour les 3-6 ans où seulement $60 \%$ des diètes étaient optimisables. Si le modèle avait permis

l'introduction de produits enrichis, le taux de faisabilité aurait été probablement plus important, comme cela a été montré avec les laits de croissance dans une population britannique de plus jeunes enfants (12-18 mois) [40].

294 L'approche de modélisation de diètes individuelles chez les SL-Excès-optimisables a montré que les changements alimentaires nécessaires à l'atteinte des recommandations sont similaires chez les enfants et les adultes (figure 2). Ainsi, l'optimisation se traduit par une augmentation de la quantité de fruits et légumes (+91 à $+236 \mathrm{~g} / \mathrm{j}$, soit 1 à 3 portions selon la classe d'âge), et de féculents $(+77$ à $+134 \mathrm{~g} / \mathrm{j}$, soit environ 1 portion). L'optimisation a diminué les boissons sucrées et les jus de fruits (pour les 2 sous-groupes : de $-69,2 \mathrm{~g} / \mathrm{j}$ à $-140,7 \mathrm{~g} / \mathrm{j}$ soit une réduction de $-0,5$ à $-0,9$ portion par jour, 
que nutriment), proposée par l'EFSA, qui est notamment très élevée pour les 14-18 ans puisqu'elle est équivalente à celle des adultes ( $2 \mathrm{~L}$ et $2,5 \mathrm{~L}$ pour les femmes et les hommes, respectivement) [33]. Or, les enfants français sont encore éloignés de cette recommandation [41], tout comme les enfants du Royaume Uni [42], des Etats-Unis [43] ou de 11 autres pays du monde [44].

L'optimisation aboutit également à une légère augmentation de la quantité de poissons chez les enfants et les adultes, alors que les quantités de viandes sont diminuées chez les adultes et augmentées chez les 3-17 ans (supplément 3 disponible en ligne). Cette différence entre adultes et enfants s'explique par une recommandation en protéines un peu plus élevée chez les enfants, alors que l’apport énergétique est plus faible que chez les adultes et que le modèle est iso-calorique.

Le groupe des produits sucrés se trouve diminué par l'optimisation dans toutes les classes d'âge (-41 à $-77 \mathrm{~g} / \mathrm{j}$ ) et cette baisse concerne tous les aliments de ce groupe. Le groupe des produits laitiers se trouve augmenté chez les 7-11 ans $(+49 \mathrm{~g} / \mathrm{j})$ et les $12-17$ ans $(+125 \mathrm{~g} / \mathrm{j})$, reste stable chez les adultes et diminué chez les 3-6 ans (-37g/j). Ces variations sont expliquées par l'augmentation des laits et yaourts nature chez les $7-11$ ans $(+57 \mathrm{~g} / \mathrm{j}$ et $+8 \mathrm{~g} / \mathrm{j}$ respectivement), les $12-17 \mathrm{ans}(+99 \mathrm{~g} / \mathrm{j}$ et $+6 \mathrm{~g} / \mathrm{j}$ respectivement) et les adultes $(+10 \mathrm{~g} / \mathrm{j}$ et $+7 \mathrm{~g} / \mathrm{j}$ respectivement), alors que la consommation de fromages est fortement diminuée quelle que soit la tranche d'âge et tout particulièrement chez les 36ans (-17 g/j) (figure 3). Dans cette tranche d'âge, les diètes sont optimisables pour seulement $60 \%$ des SL-Excès et concernent des enfants ayant déjà dans leurs diètes observées des apports élevés en calcium ( $1^{\mathrm{er}}$ quartile de la distribution supérieur à l'ANC) et des consommations importantes de produits laitiers ( $378 \mathrm{~g} / \mathrm{j}$ en moyenne). $A$ contrario, les SL-Excès dont la diète n'est pas optimisable (40\% de l'effectif des SL-Excès de 3-6 ans) présentent, en comparaison des SL-Excès optimisables, des apports faibles en calcium ( $505 \mathrm{mg} / \mathrm{j}$ versus $728 \mathrm{mg} / \mathrm{j}$ ), des niveaux de consommation plus faibles en produits laitiers ( $232 \mathrm{~g} / \mathrm{j}$ versus $378 \mathrm{~g} / \mathrm{j}$ ), et une moins bonne qualité de diète (MAR : 84 versus 91\%) (résultats non montrés). 
L'augmentation plus forte des laits nature par rapport aux yaourts nature chez les 7-17 ans peut surprendre car ces aliments ont un profil nutritionnel similaire. Elle peut s'expliquer d'une part, par la

330 fonction objectif du modèle qui privilégie les aliments consommés en grandes quantités, et d'autre part, par la teneur en eau du lait un peu plus importante que celle des yaourts, la recommandation d’apport en eau étant difficile à respecter (particulièrement chez les enfants).

De manière générale, les changements alimentaires induits par les modélisations sont en accord avec les recommandations du Programme National Nutrition Santé qui encourage la consommation de fruits et légumes, de féculents et de produits laitiers, et limite la consommation de produits et boissons sucrés, l'eau étant la seule boisson recommandée à volonté [45].

La répartition entre les différents types de sucres (totaux, libres, non libres) dans les diètes optimisées est similaire pour toutes les classes d'âge (figure 4). Les sucres totaux sont diminués dans les diètes modélisées par rapport aux diètes observées, cette diminution étant la conséquence d’une diminution des sucres libres, compensée partiellement par une augmentation des sucres non-libres (elle-même due principalement à l'augmentation des fruits et légumes). La réduction des sucres libres (-26 à $-30 \mathrm{~g} / \mathrm{j}$, selon la classe d'âge) est obtenue principalement par une diminution dans le groupe des produits sucrés (de -14,3 à -21,3g de sucres libres/j) et dans celui des boissons sucrées et jus de fruits (-6,7 à -13,4 g de sucres libres/j) qui sont les deux plus gros contributeurs en sucres libres dans les diètes observées. En revanche, dans le groupe des produits laitiers (troisième contributeur des sucres libres avec les laits aromatisés, yaourts et fromages frais sucrés), une moindre diminution des sucres libres (-0,7 à $-2 \mathrm{~g} / \mathrm{j}$ selon la classe d'âge) voire chez les $12-17$ ans, une augmentation de 2,6 $\mathrm{g} / \mathrm{j}$ des sucres libres est observée après modélisation. Ceci peut s'expliquer par le fait que les diètes modélisées doivent respecter en plus de la limite maximale en sucres libres, un ensemble de 32 
et apportant des nutriments favorables (minéraux, vitamines...) ne sont pas systématiquement

354 diminués. Par exemple, bien que les produits laitiers sucrés contiennent des sucres libres, ils

355 contribuent de manière non négligeable aux apports en calcium.

357 Le principe de la modélisation est de trouver les combinaisons d'aliments qui permettent de respecter les contraintes nutritionnelles, en s'éloignant le moins possible de la diète observée (en aliments et en poids). Aujourd'hui, cette approche théorique est largement utilisée pour répondre de manière objective à des questions de santé publique [46]. La principale force de la modélisation des diètes individuelles par rapport à la modélisation d'une diète moyenne est l'extrême diversité des solutions obtenues, puisque il existe autant de diètes respectant la totalité des contraintes nutritionnelles (33 dans cette étude) qu'il existe de diètes individuelles optimisables dans un échantillon donné. Cette approche comporte cependant certaines limites comme une intégration partielle de l'acceptabilité et des préférences alimentaires de l'individu ou la non prise en compte des moments de consommation, des associations d'aliments (ex: pain et beurre) ou encore de la consommation en portion pour certains aliments. La faisabilité ou non d'obtenir une solution mathématique résulte de la compatibilité entre les contraintes nutritionnelles, les contraintes d'acceptabilité et le répertoire alimentaire des individus. Ainsi, d'autres choix méthodologiques dans la construction du modèle (par exemple l'autorisation des laits de croissance chez des non

371 consommateurs) auraient conduit à des résultats différents, notamment sur le pourcentage de diètes

372 optimisables [40].

373 Les résultats de la modélisation individuelle, exprimés en moyenne sur les échantillons d'enfants et 374 d'adultes sont globalement cohérents avec les repères de consommation du PNNS. Cependant, les 375 changements en types et quantités d'aliments sont différents d'un individu à l'autre et aboutissent à 376 des diètes optimisées différentes les unes des autres, ce qui montre qu'il y a mille et une façons 377 alimentaires d'atteindre l'équilibre nutritionnel. 
379 En France, le pourcentage d'individus forts consommateurs de sucres libres est beaucoup plus élevé 380 chez les enfants que chez les adultes et une forte majorité des enfants a des apports excessifs en 381 sucres libres. Les diètes des enfants et adultes forts consommateurs de sucres libres sont de moins 382 bonne qualité nutritionnelle, mais peuvent généralement être optimisées (en s'éloignant le moins 383 possible de la diète observée) par une augmentation notable de la consommation de fruits, légumes, 384 féculents et eau et par une diminution significative de produits sucrés, boissons sucrées et jus de 385 fruits. Néanmoins, compte tenu des contraintes imposées dans le modèle, une exploration 386 complémentaire est nécessaire pour comprendre les raisons pour lesquelles il est impossible 387 d'atteindre une diète nutritionnellement adéquate pour $40 \%$ des enfants de 3-6 ans ayant des 388 apports excessifs en sucres libres. 
391 MS-Nutrition a obtenu une contribution financière de la part de Danone Produits Frais France pour la 392 conduite de cette étude et la rédaction de cet article. MM et LP sont salariés de MS-Nutrition. AL et 393 SV sont salariées de Danone Nutricia Research. ND n'a pas été rémunérée pour ce travail.

394 
396 [1] Moynihan PJ, Kelly S a. M. Effect on caries of restricting sugars intake: systematic review to inform WHO guidelines. J Dent Res 2014;93:8-18.

[2] Te Morenga L, Mallard S, Mann J. Dietary sugars and body weight: systematic review and meta-analyses of randomised controlled trials and cohort studies. BMJ 2013;346:e7492.

[3] Sonestedt E, Overby NC, Laaksonen DE, Birgisdottir BE. Does high sugar consumption exacerbate cardiometabolic risk factors and increase the risk of type 2 diabetes and cardiovascular disease? Food Nutr Res 2012;56.

403

[4] Greenwood DC, Threapleton DE, Evans CEL, Cleghorn CL, Nykjaer C, Woodhead C, et al. Association between sugar-sweetened and artificially sweetened soft drinks and type 2 diabetes: systematic review and dose-response meta-analysis of prospective studies. Br J Nutr 2014;112:725-34.

[5] Johnson RK, Appel LJ, Brands M, Howard B V, Lefevre M, Lustig RH, et al. Dietary Sugars Intake and Cardiovascular Health. Circulation 2009;120:1011-20.

[6] Prasad K, Dhar I. Oxidative stress as a mechanism of added sugar-induced cardiovascular disease. Int J Angiol Off Publ Int Coll Angiol Inc 2014;23:217-26.

411 [7] Morenga LA Te, Howatson AJ, Jones RM, Mann J. Dietary sugars and cardiometabolic risk: systematic review and meta-analyses of randomized controlled trials of the effects on blood

[8] Louie JCY, Tapsell LC. Association between intake of total vs added sugar on diet quality: a systematic review. Nutr Rev 2015;73:837-57. Joint WHO/FAO Expert Consultation. vol. 916. 2003. 
[10] World Health Organization. WHO Guideline: Sugars intake for adults and children. Geneva: World Health Organization; 2015.

[11] EFSA Panel on Dietetic Products Nutrition and Allergies (NDA). Scientific Opinion on Dietary Reference Values for carbohydrates and dietary fibre. EFSA J 2010;8:1462.

[12] ANSES. Avis de l'Anses relatif à l'établissement de recommandations d'apport en sucres. 2016.

[13] The Confederation of the Food and Drink Industry of the EU (CIAA). CIAA Approach to Guideline Daily Amounts (GDAs).Annex 1 to FCP/150/05E- CIAA 1. 2005.

[14] Azaïs-Braesco V, Sluik D, Maillot M, Kok F, Moreno LA. A review of total \&amp; added sugar intakes and dietary sources in Europe. Nutr J 2017;16:6.

[15] (WHO) WHO. WHO calls on countries to reduce sugars intake among adults and children. WHO 2015.

[16] Newens KJ, Walton J. A review of sugar consumption from nationally representative dietary surveys across the world. J Hum Nutr Diet 2016;29:225-40.

[17] Powell ES, Smith-Taillie LP, Popkin BM. Added Sugars Intake Across the Distribution of US Children and Adult Consumers: 1977-2012. J Acad Nutr Diet 2016;116:1543-1550.e1.

[18] Ervin R B, Ogden L. C. Consumption of Added Sugars Among U.S. Adults, 2005-2010. Natl Cent Heal Stat - Data Br 2013;122.

[19] Myhre JB, Løken EB, Wandel M, Andersen LF. The contribution of snacks to dietary intake and their association with eating location among Norwegian adults - results from a cross-sectional dietary survey. BMC Public Health 2015;15:369.

[20] Lei L, Rangan A, Flood VM, Louie JCY. Dietary intake and food sources of added sugar in the Australian population. Br J Nutr 2016;115:868-77. 
[21] Gibson S, Francis L, Newens K, Livingstone B. Associations between free sugars and nutrient intakes among children and adolescents in the UK. Br J Nutr 2016;116:1265-74.

[22] Sluik D, van Lee L, Engelen A, Feskens E. Total, Free, and Added Sugar Consumption and Adherence to Guidelines: The Dutch National Food Consumption Survey 2007?2010. Nutrients 2016;8:70.

[23] Dubuisson C, Lioret S, Touvier M, Dufour A, Calamassi-Tran G, Volatier J-L, et al. Trends in food and nutritional intakes of French adults from 1999 to 2007: results from the INCA surveys. $\mathrm{Br}$ J Nutr 2010;103:1035-48.

[24] Lluch A, Maillot M, Gazan R, Vieux F, Delaere F, Vaudaine S, et al. Individual Diet Modeling Shows How to Balance the Diet of French Adults with or without Excessive Free Sugar Intakes. Nutrients 2017;9:162.

[25] Black AE. Critical evaluation of energy intake using the Goldberg cut-off for energy intake:basal metabolic rate. A practical guide to its calculation, use and limitations. Int J Obes 2000;24:1119-30.

[26] Darmon N, Moy F. Un outil à découvrir en nutrition humaine: La programmation linèaire. Cah Nutr Diet 2008;43:303-12.

[27] Darmon N, Ferguson EL, Briend A. Impact of a cost constraint on nutritionally adequate food choices for French women: an analysis by linear programming. J Nutr Educ Behav 2006;38:8290.

[28] Masset G, Monsivais P, Maillot M, Darmon N, Drewnowski A. Diet optimization methods can help translate dietary guidelines into a cancer prevention food plan. J Nutr 2009;139:1541-8.

[29] Okubo H, Sasaki S, Murakami K, Yokoyama T, Hirota N, Notsu A, et al. Designing optimal food intake patterns to achieve nutritional goals for Japanese adults through the use of linear programming optimization models. Nutr J 2015;14:57. 
[30] Maillot M, Vieux F, Amiot MJ, Darmon N. Individual diet modeling translates nutrient recommendations into realistic and individual-specific food choices. Am J Clin Nutr 2010;91:421-30.

[31] Martin A. Apports nutritionnels conseillés pour la population Française. 3e édition. TEC\&DOC. Paris: Lavoisier; 2001.

[32] Becker W, Lyhne N, Pedersen A, Aro A, Fogelholm M, Phorsdottir I, et al. Nordic Nutrition Recommendations 2004 - integrating nutrition and physical activity. Scand J Nutr 2004;48:178-87.

[33] EFSA Panel on Dietetic Products Nutrition and Allergies (NDA). Scientific Opinion on Dietary Reference Values for water | European Food Safety Authority. EFSA J 2010;8:48.

[34] Maillot M, Vieux F, Delaere F, Lluch A, Darmon N. Dietary changes needed to reach nutritional adequacy without increasing diet cost according to income: An analysis among French adults. PLoS One 2017;12:e0174679.

[35] Guthrie HA, Scheer JC. Validity of a dietary score for assessing nutrient adequacy. J Am Diet Assoc 1981;78:240-5.

[36] Vieux F, Soler L-G, Touazi D, Darmon N. High nutritional quality is not associated with low greenhouse gas emissions in self-selected diets of French adults. Am J Clin Nutr 2013;97:56983.

[37] Swinburn BA, Caterson I, Seidell JC, James WPT. Diet, nutrition and the prevention of excess weight gain and obesity. Public Health Nutr 2004;7:123-46.

[38] Francou A, Hébel P. Le goûter en perte de vitesse et loin des recommandations. Consomm Modes Vie 2017; $\mathrm{N}^{\circ} 290$.

[39] Azaïs-Braesco V, Maillot M. Apports en sucres et principaux contributeurs dans la population 
française. Cah Nutr Diet 2017;Suppl.

489

490

491

492

493

494

495

496

497

498

499

500

501

502

503

504

505

506

507

508

509

510

[40] Vieux F, Brouzes CMC, Maillot M, Briend A, Hankard R, Lluch A, et al. Role of young child formulae and supplements to ensure nutritional adequacy in U.K. young children. Nutrients $2016 ; 8$.

[41] Vieux F, Maillot M, Constant F, Drewnowski A. Water and beverage consumption among children aged 4-13 years in France: Analyses of INCA 2 (Étude Individuelle Nationale des Consommations Alimentaires 2006-2007) data. Public Health Nutr 2016;19.

[42] Vieux F, Maillot M, Constant F, Drewnowski A. Water and beverage consumption patterns among 4 to 13-year-old children in the United Kingdom. BMC Public Health 2017;17:479.

[43] Drewnowski A, Rehm CD, Constant F. Water and beverage consumption among adults in the United States: cross-sectional study using data from NHANES 2005?2010. BMC Public Health 2013;13:1068.

[44] Iglesia I, Guelinckx I, De Miguel-Etayo PM, González-Gil EM, Salas-Salvadó J, Kavouras SA, et al. Total fluid intake of children and adolescents: cross-sectional surveys in 13 countries worldwide. Eur J Nutr 2015;54:57-67.

[45] Hercberg S, Chat-Yung S, Chaulia M. The French National Nutrition and Health Program: 20012006-2010. Int J Public Health 2008;53:68-77.

[46] Buttriss JL, Briend A, Darmon N, Ferguson EL, Maillot M, Lluch A. Diet modelling: How it can inform the development of dietary recommendations and public health policy. Nutr Bull 2014;39:115-25.

[47] Cole TJ, Lobstein T. Extended international (IOTF) body mass index cut-offs for thinness, overweight and obesity. Pediatr Obes 2012;7:284-94.

[48] Joint WHO/FAO/UNU Expert Consultation. Protein and amino acid requirements in human 
